\title{
Effect of Poling on the Mechanical Properties of $\beta$-poly(Vinylidene Fluoride)
}

\author{
C.M. Costa ${ }^{1, a}$, V. Sencadas ${ }^{1, b}$, J.F. Mano ${ }^{2,3, c}$, S. Lanceros-Méndez ${ }^{1, d}$ \\ ${ }^{1}$ Departamento de Física, Universidade do Minho, 4710-057 Braga, Portugal \\ ${ }^{2}$ Departamento de Engenharia de Polímeros, Universidade do Minho, \\ 4800-058 Guimarães, Portugal. \\ ${ }^{3} 3 \mathrm{~B}$ 's Research Group - Biomaterials, Biodegradables and Biomimetics, \\ University of Minho, 4710-057 Braga, Portugal. \\ acmscosta@portugalmail.pt, ${ }^{b}$ vsencadas@fisica.uminho.pt, ${ }^{c}$ jmano@dep.uminho.pt, \\ dlanceros@fisica.uminho.pt
}

\section{Keywords: ferroelectrics, PVDF, piezoelectric, thermal, mechanical}

\begin{abstract}
In this work, mechanical and thermal experimental techniques have been applied in order to relate the mechanical response with the microscopic variations of the material. Stress-strain results along the main directions of $\beta$-poly(vinylidene fluoride), $\beta$-PVDF, in poled and non-poled samples enables to investigate the influence of the poling process on the mechanical response of the material. Further, differential scanning calorimetry experiments allow the investigation of the effect of poling in the degree of crystallinity of the material as well as on the stability of the crystalline phase. Thermogravimetric analysis was used to investigate the kinetics of the thermal degradation of poled and non-poled $\beta$-PVDF samples. The differences observed between the two materials suggest that the poling affects the mechanical properties of the material especially in the direction parallel to the polymeric chains and originates changes at a molecular level that remain beyond the melting of the material.
\end{abstract}

\section{Introduction}

Poly(vynilidene fluoride) (PVDF) is the prototype ferroelectric polymer. A wide range of studies have been reported on PVDF and its copolymers, due to its attractive piezo- and pyroelectric properties. PVDF is a semi-crystalline polymer, exhibiting different crystalline phases. The $\beta$-form is the one that provides the best ferroelectric properties, but the $\alpha$-form is the more stable one. This phase is formed during simple crystallization upon cooling from the melt [1-3].

PVDF is a linear fluorinated hydrocarbon with a repeat unit $\left(\mathrm{CH}_{2}-\mathrm{CF}_{2}\right)$ of spacing 2.6 $\AA$. The PVDF chains have a net dipole moment nearly perpendicular to the polymer chain, pointing from the electronegative fluorine to the electropositive hydrogen. The polymer chains can crystallize in a quasi-hexagonal close-packed $\beta$-phase structure.

In order to prepare $\beta$-PVDF it is first necessary to prepare electrically inactive $\alpha$-PVDF by stretching and poling [4]. Before poling, the distribution of directions of polarisation of individual crystallites is random, whereas afterwards the polarisation directions have a biased distribution in space, and are biased along the direction of the applied electric field [4].

The poling is based on the cooperative orientation of the monomer dipoles $\left(\mathrm{CH}_{2}-\mathrm{CF}_{2}\right)$ in ferroelectric domains [5]. The $\beta$-PVDF structure is highly oriented; it has orthorhombic unit cells with a space group $\mathrm{Cm} 2 \mathrm{~m}\left(\mathrm{C}_{2 \mathrm{v}}\right)$ with a planar zig-zag (all-trans) chain conformation and parallel $\mathrm{CF}_{2}$ dipole moments of the adjacent chains parallel to the crystallographic b-axis. The conformation repeat unit has a dipole moment of $7,0 \times 10^{-30} \mathrm{Cm}$. $\beta$-PVDF has therefore a polarization of $0.13 \mathrm{C} / \mathrm{m}^{2}$ and exhibits the strongest electrical activity of all crystalline phases. An important issue is the 
dependence of those properties on the structure of PVDF. The material is composed by small crystallites with typical dimensions of $\sim 10 \mathrm{~nm}$ organised in groups of lamella, with partially oriented amorphous intralamella phase $[3,6]$. In normal ferroelectrics (FE) the domain size should be larger that a critical value; otherwise, the domain will not be stable and collapse [7]. It was proposed that the amorphous phase in FE polymers plays an important role in the formation of stable ferroelectric domains, which should contain several crystallites and part of the amorphous phase. One or more lamella could participate in each domain. In fact, it has been concluded that the interphase between the crystalline and amorphous phases is of great importance for the polarization in $\beta$-PVDF [8].

As the poling process will involve chains in the interface as well as the rotation of the dipoles in the crystallites, significant changes in the thermal and mechanical properties of the material should appear due to the poling. The purpose of this work is to investigate how the poling and the piezoelectric coupling affect the mechanical response of the material. Special attention will be paid to the variations in the degree of crystallinity and modifications at a molecular level that deeply influence the macroscopic response of the material.

\section{Experimental}

The starting material was a $28-\mu \mathrm{m}$ thick poled and unpoled commercial PVDF film from the same batch from Measurements Specialties, Incorporated (Fairfield, NJ). The Stress-Strain measurements were carried out a Minimat machine from Polymer Laboratories at $d \mathrm{l} / \mathrm{dt}=2 \mathrm{~mm} / \mathrm{min}$ on $4 \mathrm{~cm} \times 1 \mathrm{~cm}$ rectangular samples in the directions parallel and perpendicular to the preferred chain orientation at room temperature. Thermal analysis was carried out by Differential Scanning Calorimetry (DSC) with a Perkin Elmer DSC apparatus, at a heating rate of $5^{\circ} \mathrm{C} / \mathrm{min}$, under nitrogen atmosphere. The study of the chemical degradation was performed in a Perkin Elmer TGA 7, thermogravimetric analyzer, at a heating rate of $5^{\circ} \mathrm{C} / \mathrm{min}$, under nitrogen atmosphere.

\section{Theoretical considerations}

For the description of the behaviour of the stress/strain curves, it is convenient to consider the strain stress and polarization $(\varepsilon, P)$ as independent variables. In this case, the appropriate energy functional which incorporates linear electromechanical coupling is the Helmholtz relation

$$
\psi(\varepsilon, P)=\frac{1}{2} E_{i j k l}^{P} \varepsilon_{i j} \varepsilon_{k l}-b_{n i j} \varepsilon_{i j} P_{n}+\frac{1}{2} \alpha_{n m}{ }^{\varepsilon} P_{m} P_{n},
$$

where $E_{i j k l}^{P}$ denotes the Young's modulus at constant polarization, $b_{n i j}$ is a piezoelectric coupling coefficient and $\alpha_{n m}{ }^{\varepsilon}$ is the dielectric coefficient. The first and third terms on the right side of the eq.1 quantify the elastic and electrostatic energies, respectively. The second term incorporates linear contributions due to electromechanical coupling with the negative sign chosen to ensure that the coefficient has the same sign as the piezoelectric coefficient $d$.

For the case of curve stress/strain, the linear constitutive relations expressed in terms of the independent variables and $\varepsilon$ it $P$ are

$$
\begin{aligned}
& \sigma_{i j}=E_{i j k l}{ }^{P} \varepsilon_{k l}-b_{n i j} P_{n}, \\
& E_{n}=-b_{n i j} \varepsilon_{i j}+\alpha_{n m}{ }^{\varepsilon} P_{m},
\end{aligned}
$$

where $\sigma_{i j}$ is mechanical stress and $E_{n}$ is electric field vector. The piezoelectric coupling coefficient $b_{n i j}$ is related to the piezoelectric coefficient $d_{n i j}$ by:

$$
d_{n i j}^{-1}=k_{n i j} * b_{n i j}
$$


here, $k_{n i j}$ is electromechanical coupling factor [9].

\section{Results and discussion}

Figure 1 shows the stress/strain curves with stress applied along the preferred chain direction for poled and unpoled $\beta$-PVDF measured in open circuit conditions. The Young's modulus at constant polarization obtained from equation 2 for unpoled PVDF, is $0.9 \mathrm{GPa} \pm 0.1 \mathrm{GPa}$ and for poled PVDF is $1.3 \mathrm{GPa} \pm 0.1 \mathrm{GPa}$, i.e the poling process affects the mechanical properties of the material along the chain direction. This reflects the piezoelectric coupling along the stretch direction, which can be estimated from the equation 4 . The piezoelectric coupling coefficient can be thus calculated as $\mathrm{b}_{31}=158^{*} 10^{9} \mathrm{~N} / \mathrm{C}$. With the measured value of the $\mathrm{d}_{31}=23 \mathrm{pC} / \mathrm{N}$, the value of the electromechanical coupling factor $\mathrm{k}_{31}=0.27$.

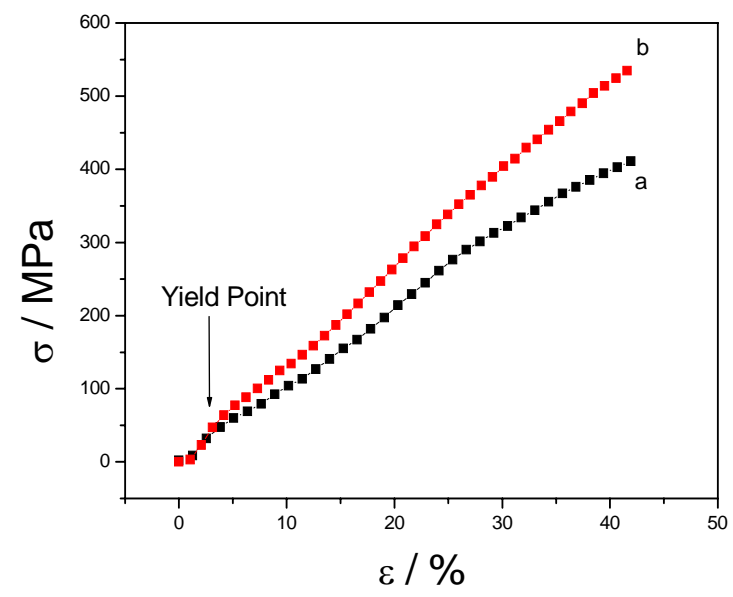

Figure 1. Stress/Strain curves for non-poled (a) and poled (b) $\beta$-PVDF

However, stress-strain curves perpendicular to the preferred chain orientation (direction 2) for poled and unpoled material show similar values in the Young modulus, yield stress and ultimate stress and strains [10]. The poling and the electromechanical coupling along this direction do not affect the macroscopic mechanical response of the material significantly.

DSC experiments for poled PVDF show a double peak structure [10] (Figure 2). This structure might indicate a coexistence $\beta$ and $\alpha$ phase and/or imperfect $\beta$ phase induced during the high temperature poling process. For upoled PVDF a single peak was obtained. From the DSC curves the degree of crystallinity $\chi(\%)$ can be calculated from the enthalpy of the melting peak $\Delta \mathrm{H}_{0} / \mathrm{Jg}^{-1}$, taken into consideration the enthalpy for a fully crystallized PVDF $\left(\Delta H_{100}=104.50 \mathrm{~J} / \mathrm{g}\right.$ for $\beta$-PVDF and $\Delta H_{100}=93.07$ for $\alpha$-PVDF) [10] (Table I). As can be observed, poling slightly decreases the degree of crystallinity $\chi(\%)$ due to the $\alpha$ to $\beta$ transformation or defective $\beta$ phase induced during high temperature.

\begin{tabular}{|c|c||r||r|r|}
\hline \multicolumn{2}{|c||}{} & \multicolumn{1}{|c|}{$\mathrm{T}_{\mathrm{f}} /{ }^{\circ} \mathrm{C}$} & \multicolumn{1}{|c|}{$\Delta \mathrm{H}_{0} / \mathrm{Jg}^{-1}$} & \multicolumn{1}{c|}{$\chi(\%)$} \\
\hline \multirow{2}{*}{ Poled } & $1^{\circ}$ run & 156.3 & 46.031 & 44.05 \\
\cline { 2 - 5 } & $2^{\circ}$ run & 161.5 & 38.956 & 41.86 \\
\hline \hline \multirow{2}{*}{ Unpoled } & $1^{\circ}$ run & 160.1 & 48.853 & 46.75 \\
\cline { 2 - 5 } & $2^{\circ}$ run & 169 & 39.672 & 42.63 \\
\hline
\end{tabular}

Table I: Main parameters obtained for the first and second heating and cooling runs for poled (above) and unpoled (below) $\beta$-PVDF. $\mathrm{T}_{\mathrm{f}}$ is the melting temperature, $\Delta \mathrm{H}_{0}$ is the melting enthalpy, and $\chi(\%)$ is the degree of crystallinity. 

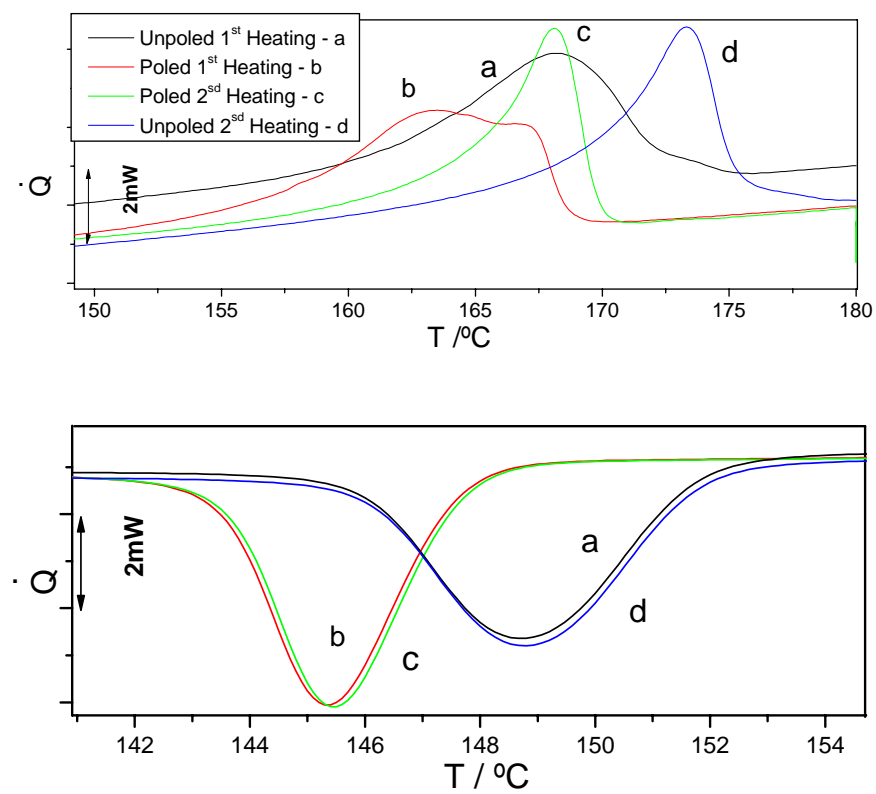

Figure 2. First and second run of the DSC scans for poled and unpoled $\beta$-PVDF. Top: heating runs; bottom: cooling runs.

After melting, the film re-crystallizes in the $\alpha$-phase. The second run for both poled and unpoled $\beta$ PVDF should be similar, as they come from the same batch. Instead of that, a variation of $\sim 9{ }^{\circ} \mathrm{C}$ in the melting temperature is found (Table I). This indicates that poling induces changes at a molecular level that remain beyond the melting of the material, and that the $\alpha$-phase obtained by recrystallization still show some effects of the poling process. This fact is further confirmed by the results of the thermo gravimetric experiments. The results of the amount of converted mass (C) and the conversion rate $(\mathrm{dC} / \mathrm{dT})$ are presented in Figure. 3, left. The poling process seems to shift the thermal degradation towards higher temperatures. This means that the effect of poling remain wide beyond the melting transition of the material, i.e. changes at a molecular level are induced and preserved over the wide temperature range.
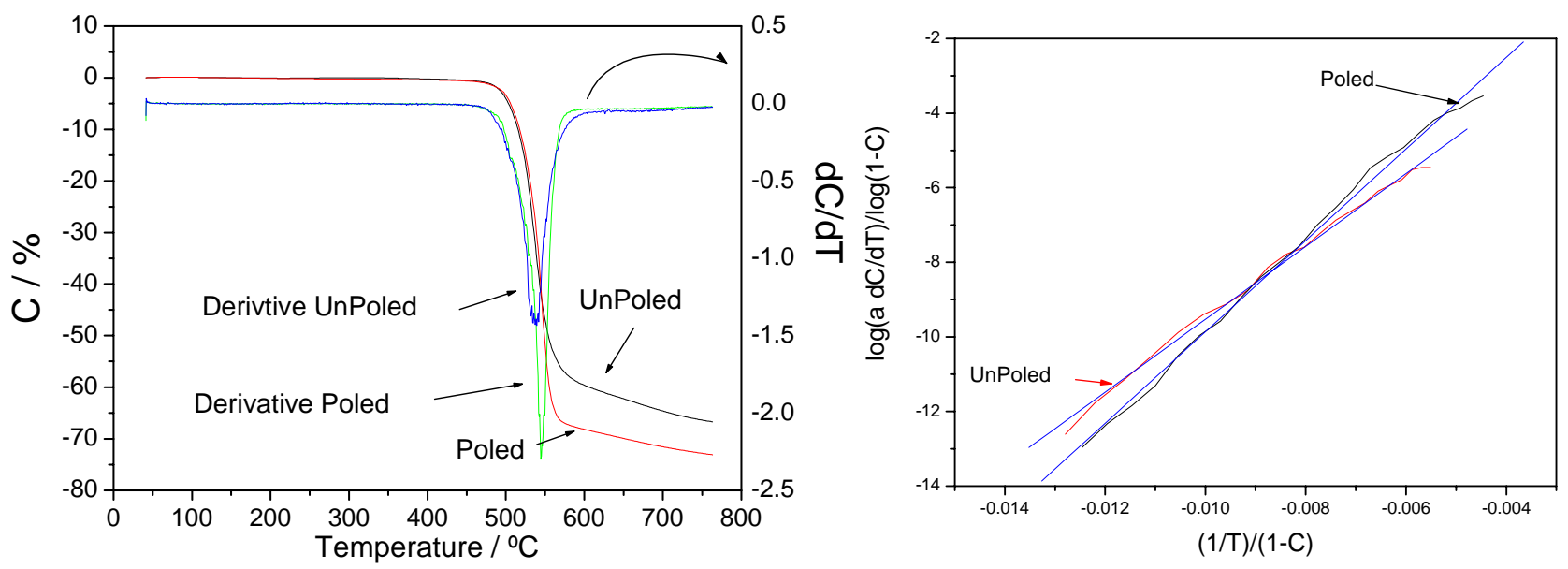

Figure 3. Left: Thermogravimetric results in poled and non-poled $\beta$-PVDF and the corresponding derivatives. $\mathrm{C}$ is the amount of converted mass, in $\%$ and $\mathrm{dC} / \mathrm{dT}$ is the conversion rate. Right: Calculation of the activation energy. 
In order to evaluate the activation energy of the thermal degradation, the conversion rate of mass with time $t$ can be expressed as follows [11]:

$$
d C / d t=-k C^{n} \exp [-E / R T(t)](n \geq 1),
$$

where $n$ is the order of the reaction, $E$ is the activation energy of the process, $T$ is the temperature, $C$ is the $\%$ of the loosed mass $R$ is the universal gas constant and $t$ is the time. In a constant heating rate the equation 6 can be written as:

$$
a \frac{d C}{d T}=(1-C)^{n} \exp [-E / R T]
$$

Here, $a$ is the heating rate, and $d C / d T$ is the conversion rate and $R$ is the universal gas constant. By a linear regression of the results of Figure 3 (left) with equation 6 (Figure 3, right) for poled and unpoled PVDF the activation energy of the degradation process can be estimated. The results obtained for poled and non-poled PVDF are $E=23 \mathrm{~kJ} / \mathrm{mol}$ and $E=18.7 \mathrm{~kJ} / \mathrm{mol}$, respectively, i.e. the activation energy is larger for the poled material. The excess of energy is probably due to excess of energy due to the poling process.

\section{Conclusions}

The influence of the poling on the degree of crystallinity and mechanical response of the material has been discussed by analyzing the mechanical and thermal response of poled and unpoled $\beta$ PVDF. As a result, the Young modulus of the material along the polymeric chains increases but does not especially affect the mechanical properties in the transversal direction, i.e. poling mainly affects the intrachain coupling. Further, poling not only induces a rotation of the dipolar moments towards the direction of the applied field, but also causes modifications in the crystalline structure and at a molecular level [12]. This is confirmed by the differences found between the poled and unpoled material found in the first and second runs of the DSC experiments as well as in the thermal degradation temperatures and energies obtained in thermogravimetric experiments.

\section{References}

[1] Blinov LM, Fridkin VM, Palto SP, Bune AV, Downen PA, Durcharme S, Physiscs-Uspekhi Vol. 43 (2000), p. 243

[2] Furukawa T Phase Transitions Vol, 18 (1989), p. 143

[3] Lovinger AJ, in Developments in Crystalline Polymers, edited by I.D.C. Basset, Elsevier Applied Science, London, 1982

[4] Dvey-Aharon H, Sluckin TJ, et al., Phys Rev. B, Vol. 21(1980), p. 3700

[5] Yamada T, Ueda T, Kitayama T J. App. Phys. Vol. 52 (1981), p.948.

[6] Lovinger AJ Science Vol. 220 (1983), p. 1115

[7] Strukov BA, Levanyuk AP, Ferroelectric Phenomena in Crystals: Physical Foundations, Springer-Verlag, Berlin, 1998.

[8] Harnischfeger P, Jungnickel B-J Ferroelectrics, Vol. 109 (1990), p. 279

[9] Ikeda T, Fundamentals of Piezoelectricity; Oxford Uiversity Press, 1990

[10] Sencadas V, Lanceros-Mendez S, Mano JF, Thermochimica acta Vol 424 (2004), p. 201

[11] Pawlak A, Mucha M, Thermochimica Acta, Vol. 396 (2003), p. 153.

[12] Sencadas V, Costa CM, Moreira V, Monteiro J, Mendiratta SK, Mano, JF and LancerosMendez, S, e-Polymers (2005), nº 002 